\title{
Parámetros Reproductivos de Llambina Potamorhina altamazonica (Characiformes: Curimatidae) en el Río Ucayali
}

\author{
Reproductive Parameters of Llambina Potamorhina altamazonica (Characiformes: \\ Curimatidae) in the Ucayali River
}

\author{
Stive Flores-Gómez ${ }^{1,2}$
}

\section{Resumen}

La llambina Potamorhina altamazonica es la segunda especie dominante en los desembarques de la pesquería comercial en la región Ucayali, y de gran importancia en la socioeconomía regional. En el presente estudio se determinaron las características reproductivas de la llambina: periodo de desove, proporción sexual, talla media de primera madurez y factor de condición. El análisis mostró que el desove guarda sincronía con el incremento del nivel del agua del río Ucayali en la época de transición a creciente y en la creciente, que inicia en octubre, con intenso desove entre enero y febrero, y culmina en marzo. La proporción sexual fue significativamente favorable a las hembras en la mayoría de las épocas de año. La talla a la primera madurez sexual se estimó en $17.8 \mathrm{~cm}$ en hembras y $18.4 \mathrm{~cm}$ en machos (longitud total). Los peces mostraron mejor condición somática en la época de vaciante (julio a octubre), que corresponde al periodo en que el detrito es de mejor calidad. Los resultados muestran que la especie tiene una estrategia de vida estacional o periódica (r2), cuyo ciclo reproductivo está estrechamente relacionado con los cambios hidrológicos del río.

Palabras clave: Amazonia, estacional, estrategia de vida, desove, condición somática

\section{ABstRACT}

«Llambina» Potamorhina altamazonica is the second dominant species in the commercial fishery landings in the Ucayali region and of great importance in the regional socio-economics. The aim of this study was to determine the reproductive characteristics of this species. Spawning period, sex ratio, average size at maturity and condition factor were analyzed. The analysis showed that the spawning have synchrony with the increase

\footnotetext{
${ }^{1}$ Área de Investigaciones de Recursos en Aguas Continentales, Instituto del Mar del Perú. Callao, Perú

${ }^{2}$ E-mail: danstive@gmail.com
}

Recibido: 10 de setiembre de 2014

Aceptado para publicación: 29 de noviembre de 2014 
of water level in the Ucayali river in the transition to high water and in the high water seasons, starting in October, with intense spawning between January and February and completion in March. The sex ratio was significantly favorable to females at most times of the year. The average size at first sexual maturity was estimated at $17.8 \mathrm{~cm}$ in females and $18.4 \mathrm{~cm}$ in males (total length). The fish showed better somatic condition in the dry season (July to October), which corresponds to the period when the detritus is of better quality. The results show that llambina has a seasonal or periodic strategy of life (r2), and the reproductive cycle is closely related to river hydrological changes.

Key words: Amazon, seasonal, life strategy, spawning, somatic condition

\section{INTRODUCCIÓN}

Las estadísticas de desembarque de la flota pesquera comercial de Pucallpa, Perú, muestran que la llambina Potamorhina altamazonica, después del boquichico Prochilodus nigricans, es la segunda especie con los más altos volúmenes de desembarque, representando más del $17 \%$ de las capturas durante el periodo 1994-2006 en la región Ucayali (Riofrío et al., 2008).

La llambina es una especie bentopelágica que habita en los márgenes de los lagos y ríos de agua blanca o negra en la cuenca del río Amazonas (Yamamoto, 2004). Es detritívora, posee dieta constituida de materia orgánica, algas y microorganismos que viven en la lama (Pouilly et al., 2004). Es de hábito diurno, comportamiento migratorio, desove total y fecundación externa; en la época de reproducción forma grandes cardúmenes para desovar en ríos de agua blanca (Lima y Araújo-Lima, 2004).

El escaso conocimiento de la biología de las especies acuáticas en la Amazonia peruana es una limitante para que se adopten correctas medidas de conservación y uso sostenible, siendo conveniente resaltar que es un recurso de importancia porque contribuye con la alimentación y economía regional (Guerra, 1995). Las investigaciones en peces teleósteos que habitan en aguas continentales tropicales de Sudamérica evidencian que varias especies han adoptado una diversidad de estrategias reproductivas. El desove de varias de ellas está relacionada a las condiciones ambientales, siendo el régimen hidrológico y, más específicamente, el pulso de inundación, uno de los factores que ejerce influencia sobre la reproducción, principalmente en aquellas especies que han desarrollado una estrategia de vida del tipo estacional (Winemiller, 1989; Guerrero et al., 2009).

El objetivo del presente estudio fue determinar el periodo de desove, proporción sexual, talla media de primera madurez y factor de condición de Potamorhina altamazonica en el río Ucayali.

\section{Materiales y Métodos}

Se obtuvieron muestras biológicas mensuales de los desembarques de la flota pesquera comercial en el puerto de Pucallpa, Perú (551899.46 m E, $9072670.78 \mathrm{~m} \mathrm{~S}$ UTM) entre 2008 y 2012. Se registró el lugar de procedencia del material biológico, determinando que las muestras provinieron principalmente de Callería, Utiquinia, Abujao, Tamaya, Shesha, Iparía y Aruya, subcuencas que se encuentran dentro del ámbito geográfico de la región Ucayali (Fig. 1).

Para el análisis de información se consideraron los registros de niveles del agua del río Ucayali, tomados en la estación La Hoyada (Pucallpa), por la Dirección Regional de Transporte Acuático de Ucayali, entre 2008 y 2012. 


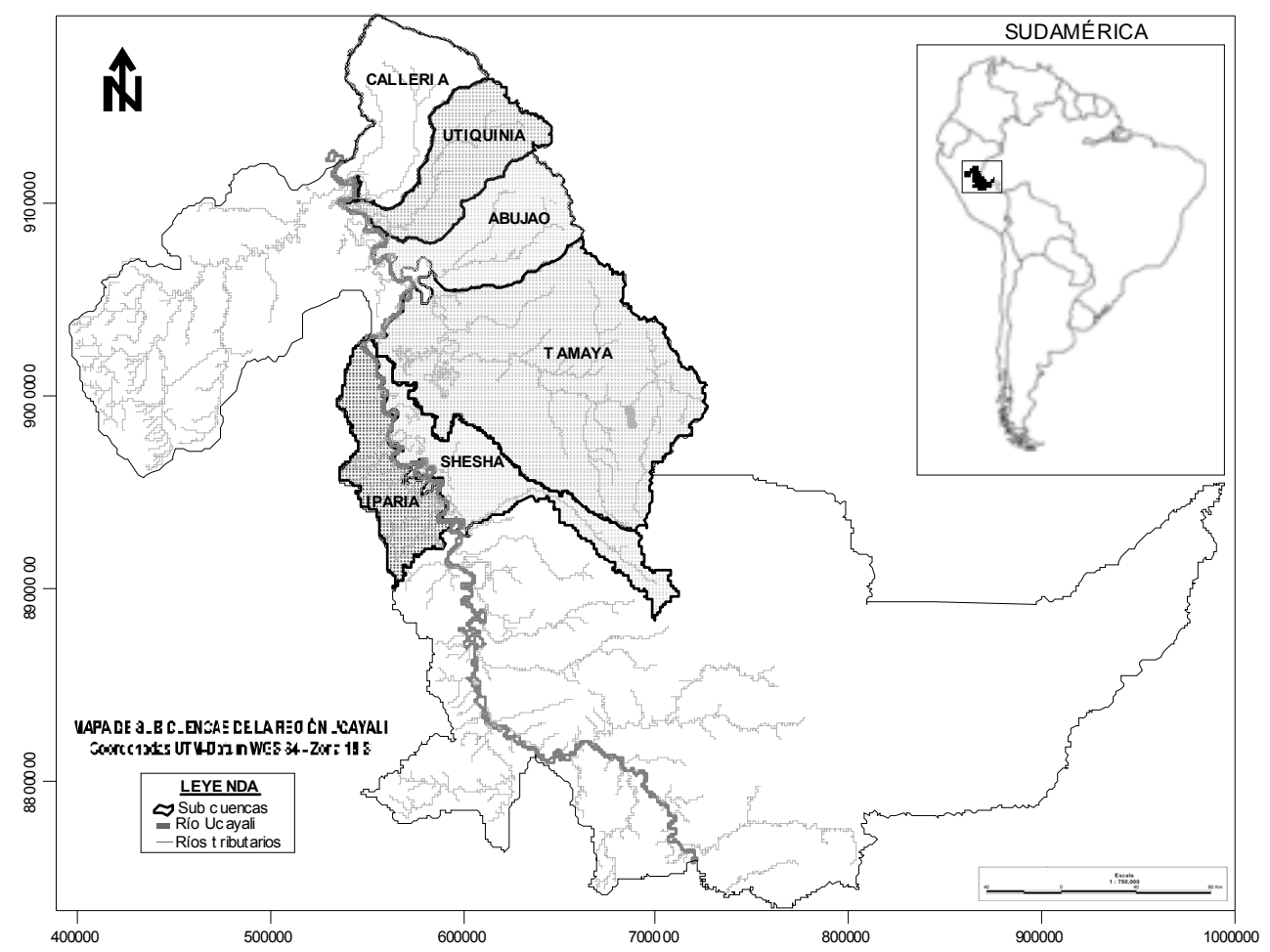

Figura 1. Mapa de subcuencas de la región Ucayali. Los nombres corresponden a las zonas de procedencia del material biológico analizado. Imagen editada a partir del mapa de subcuencas de la región Ucayali - PROYECTO UCRU (2010)

En el muestreo biológico mensual se observaron no menos de 10 individuos por clase de talla. En cada individuo se registró la longitud total (LT), longitud a la horquilla (LH) y longitud estándar (LE) en centímetros, los pesos total (PT) y eviscerado (PE) en gramos, sexo, estadio de madurez gonadal [empleando la escala propuesta por Flores (2013)] y el peso gonadal (g).

La longitud de cada pez fue medida con un ictiómetro calibrado a $0.5 \mathrm{~cm}$, el peso total con una balanza con $0.1 \mathrm{~g}$ de precisión y el peso de las gónadas con una balanza de $0.01 \mathrm{~g}$ de precisión. La proporción sexual fue analizada por periodos hidrológicos (acumulando en cada periodo la información de los años 2008-2012) del río Ucayali. La propor- ción sexual teórica $1 \mathrm{H}$ : $1 \mathrm{M}$ fue analizada aplicando la prueba estadística de Chi cuadrado $(\alpha=0.05)$, considerándose como significativo a $\mathrm{X}^{2} \geq 3.84$ (valor de tabla: 1 grados de libertad).

El periodo de desove fue determinado por el análisis de la evolución mensual multianual del índice gonadosomático (IGS), según el método de Vazzoler (1996), VillamilMoreno y Arias-Castellano (2011), y por el análisis de la progresión mensual de estadios de madurez gonadal, según García et al. (2010). La ecuación es IGS $=\mathrm{P}_{\mathrm{G}} / \mathrm{P}_{\mathrm{E}} \times 100$; donde: $\mathrm{P}_{\mathrm{G}}$ es el peso de las gónadas $(\mathrm{g}) \mathrm{y}$ el $\mathrm{P}_{\mathrm{E}}$ el peso eviscerado ( $\mathrm{g}$ ) de cada espécimen (Buitrón et al., 2011). 
Cuadro 1. Ecuaciones de regresiones lineales longitud total $\left(\mathrm{L}_{\mathrm{T}}\right)$, longitud a la horquilla $\left(\mathrm{L}_{\mathrm{H}}\right)$ y longitud estándar $\left(\mathrm{L}_{\mathrm{E}}\right)$ de llambina Potamorhina altamazonica del río Ucayali

\begin{tabular}{llll}
\hline $\begin{array}{l}\text { Tipo de } \\
\text { longitud }\end{array}$ & $\mathrm{L}_{\mathrm{T}}$ & $\mathrm{L}_{\mathrm{H}}$ & $\mathrm{L}_{\mathrm{E}}$ \\
\hline $\mathrm{L}_{\mathrm{T}}$ & & $\mathrm{L}_{\mathrm{H}}=0.865 \mathrm{~L}_{\mathrm{T}}+0.841$ & $\mathrm{~L}_{\mathrm{E}}=0.809 \mathrm{~L}_{\mathrm{T}}+0.133$ \\
& & $\mathrm{R}^{2}=0.959$ & $\mathrm{R}^{2}=0.941$ \\
$\mathrm{~L}_{\mathrm{H}}$ & $\mathrm{L}_{\mathrm{T}}=1.109 \mathrm{~L}_{\mathrm{H}}-0.063$ & & $\mathrm{~L}_{\mathrm{E}}=0.930 \mathrm{~L}_{\mathrm{H}}-0.548$ \\
& $\mathrm{R}^{2}=0.959$ & & $\mathrm{R}^{2}=0.969$ \\
$\mathrm{~L}_{\mathrm{E}}$ & $\mathrm{L}_{\mathrm{T}}=1.162 \mathrm{~L}_{\mathrm{E}}+1.111$ & $\mathrm{~L}_{\mathrm{H}}=1.042 \mathrm{~L}_{\mathrm{E}}-1.166$ & \\
& $\mathrm{R}^{2}=0.941$ & $\mathrm{R}^{2}=0.969$ & \\
\hline
\end{tabular}

Cuadro 2. Variación estacional de la proporción sexual de la llambina Potamorhina altamazonica en el río Ucayali (2008-2012)

\begin{tabular}{lccccc}
\hline & $\begin{array}{c}\text { Hembra } \\
(\mathrm{n})\end{array}$ & $\begin{array}{c}\text { Macho } \\
(\mathrm{n})\end{array}$ & $\begin{array}{c}\text { Total } \\
(\mathrm{n})\end{array}$ & $\begin{array}{c}\text { Proporción } \\
(\mathrm{H}: \mathrm{M})\end{array}$ & $\mathrm{X}^{2}$ \\
\hline Creciente & 629 & 396 & 1025 & $1.6: 1.0$ & $53.0^{*}$ \\
Transición a vaciante & 680 & 566 & 1246 & $1.2: 1.0$ & $10.4^{*}$ \\
Vaciante & 493 & 475 & 968 & $1.0: 1.0$ & 0.3 \\
Transición a creciente & 842 & 933 & 1775 & $0.9: 1.0$ & $4.7^{*}$ \\
\hline Total & 2644 & 2370 & 5014 & $1.1: 1.0$ & $15.0^{*}$ \\
\hline
\end{tabular}

$* x^{2}=3.84 ; p<0.05$

Se analizó la relación entre el IGS y el nivel del agua del río Ucayali mediante una regresión lineal $\mathrm{Y}=b \mathrm{X}+a$ (donde $b$ es la pendiente y $a$ es el intercepto) y se determinó el coeficiente de determinación $\left(\mathrm{r}^{2}\right)$ entre los puntos respecto a la pendiente.

La talla media de primera madurez $\left(\mathrm{L}_{50}\right)$ fue estimada según los términos de Cubillos (2005), quien define $\mathrm{L}_{50}$ como la talla en la ojiva de madurez en la cual existe $50 \%$ de probabilidad de observar un individuo maduro, expresando la siguiente ecuación $\mathrm{P}_{\mathrm{L}}$ $=1 / 1+\mathrm{e}^{\alpha+\beta \mathrm{L}}$; donde: $\alpha$ y $\beta$ son constantes $\mathrm{y}$
$\mathrm{P}_{\mathrm{L}}$ es la proporción de hembras maduras a la talla L (cm). La talla media de primera madurez sexual fue definida por $\mathrm{L}_{50}=\alpha / \beta$. Se utilizó la información biológica de los meses dentro de la época de reproducción de los años 2008 y 2012 y la media por combinación de ambos (no se pudo estimar para los años 2009 a 2011 por información deficiente). Los valores de $\mathrm{L}_{50}$ entre sexos y años fueron comparados usando la prueba $t$ Student (grados de libertad calculados del número de clase de longitudes), utilizando un valor $\alpha=0.05$. 


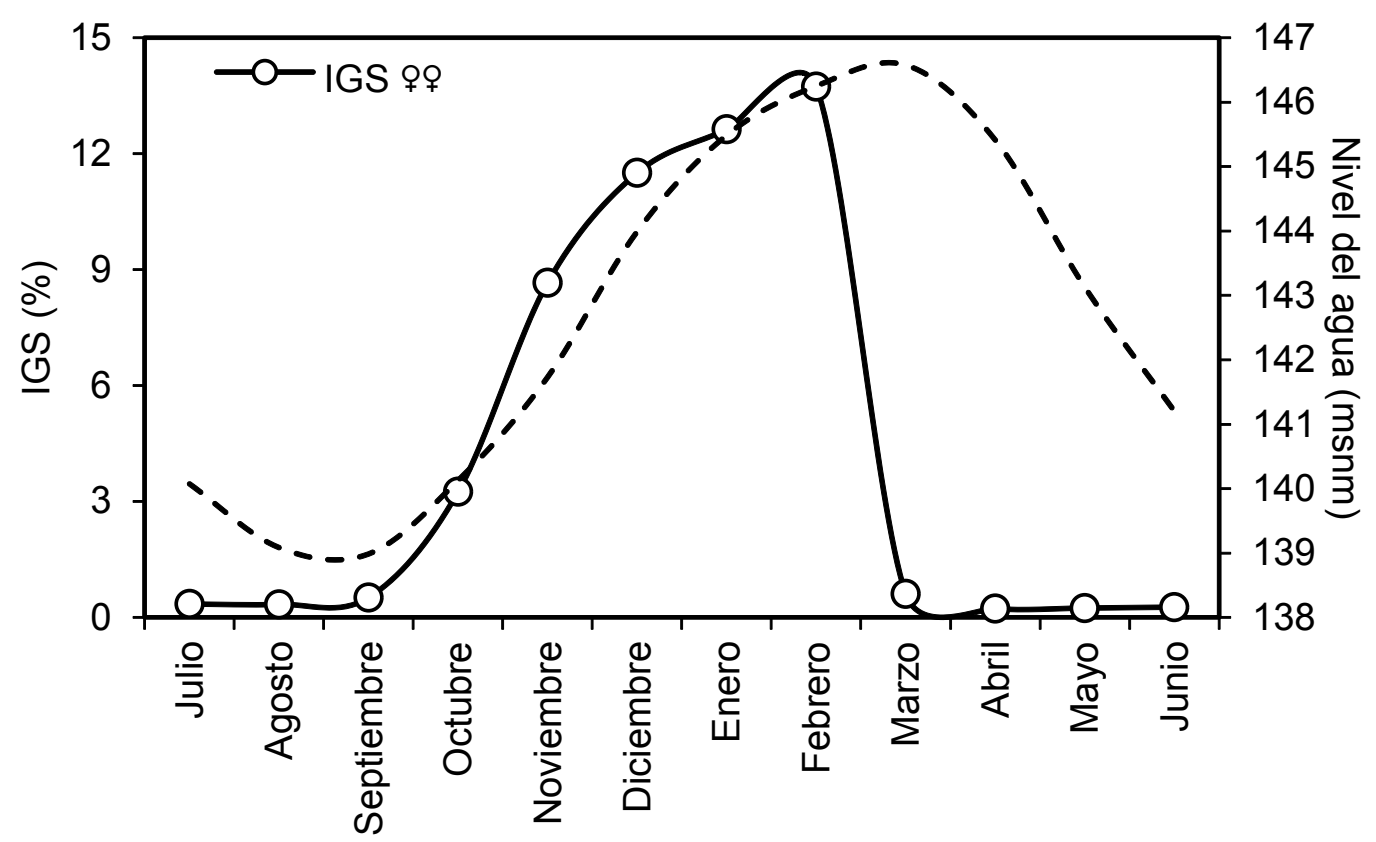

Figura 2. Variación mensual del índice gonadosomático (0) de hembras de Potamorhina altamazonica $(\mathrm{n}=2644)$ y del nivel de agua del río Ucayali $(-)(2008-2012)$

Se analizó la variación temporal del factor de condición de Fulton $(\mathrm{K})$, que viene dada por la ecuación $\mathrm{K}=\mathrm{P}_{\mathrm{E}} / \mathrm{L}_{\mathrm{T}}^{3}$ x 100; donde $\mathrm{L}_{\mathrm{T}}$ es la longitud total y $\mathrm{P}_{\mathrm{E}}$ es el peso eviscerado del pez. Se empleó $\mathrm{P}_{\mathrm{E}}$ a fin de evitar la interferencia del peso de la gónada y del estómago sobre K.

Con la información de la longitud total $\left(\mathrm{L}_{\mathrm{T}}\right)$, longitud a la horquilla $\left(\mathrm{L}_{\mathrm{H}}\right)$ y longitud estándar $\left(\mathrm{L}_{\mathrm{E}}\right)$, se determinó la relación existente entre ellas con el fin de obtener ecuaciones que permitan realizar conversiones de una longitud a otra (Cuadro 1).

\section{Resultados}

Se observaron 5014 ejemplares en el periodo de estudio. La proporción sexual global fue significativamente mayor en las hembras (Cuadro 2); sin embargo, al hacer el análisis estacional, se encontró que en el periodo de transición a creciente hubo dominancia de machos $(\mathrm{p}<0.05)$, mientras que en la creciente y transición a vaciante dominaron las hembras $(\mathrm{p}<0.05)$. En la vaciante no hubo diferencias significativas entre sexos (Cuadro 2).

\section{Periodo de Desove}

Los valores mayores de $3 \%$ en hembras y $0.1 \%$ en machos en el análisis de la evolución mensual del índice gonadosomático (IGS) permiten determinar que la reproducción de la especie en estudio acontece entre los meses de octubre y marzo, alcanzando el pico en febrero para el caso de las hembras (14\%); mientras que los valores de IGS estuvieron bajos ( $<1 \%$ en hembras) entre marzo y setiembre (Fig. 2).

La incidencia de hembras en desove (Fig. 3) fue representativa entre los meses de setiembre (22.5\%) y marzo (9.9\%), superando el $60 \%$ entre noviembre y febrero, con el pico en enero (95.7\%). Se observó una relación directa entre el incremento del IGS y el nivel agua del río (Fig. 4) entre setiembre 


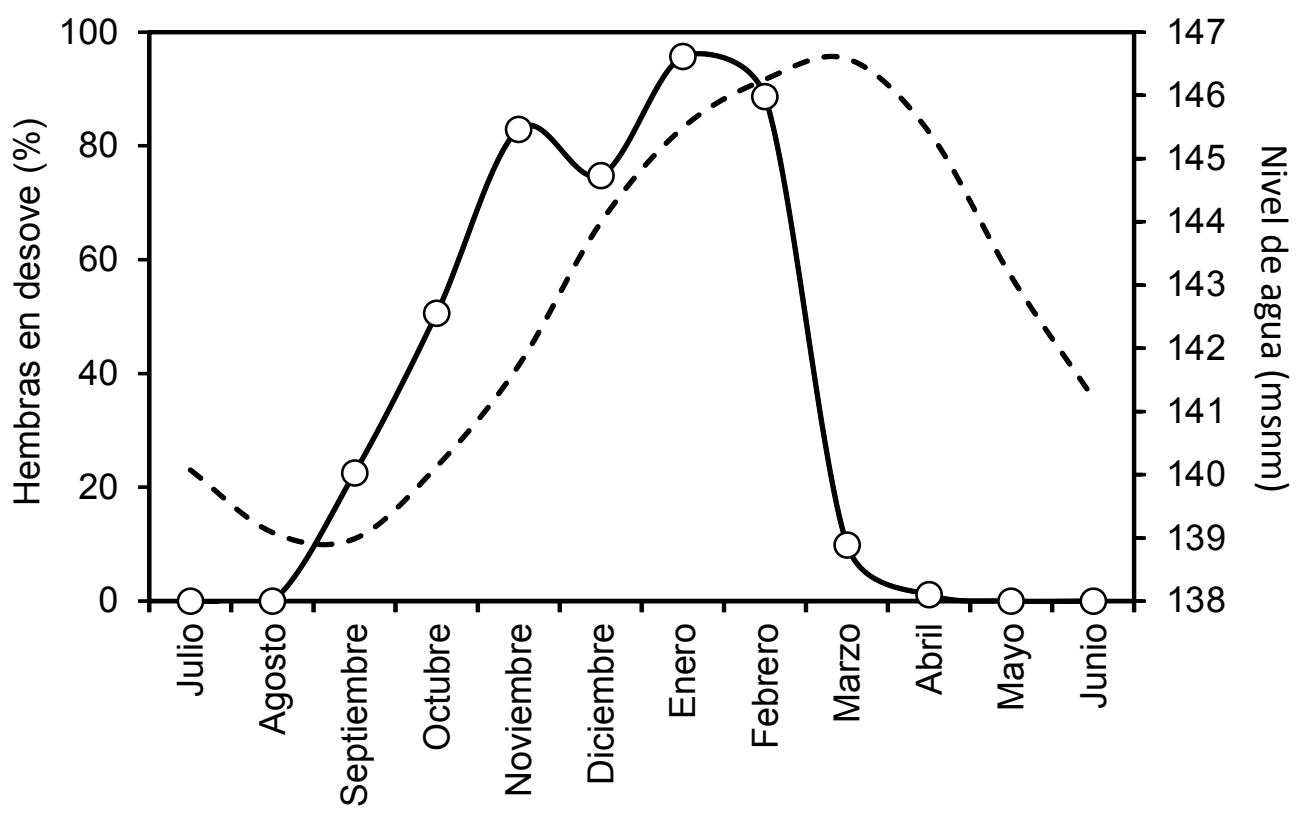

Figura 3. Variación mensual del porcentaje de hembras de Potamorhina altamazonica en desove (estadios 4 y 5) (0) y del nivel de agua del río Ucayali (-) (2008-2012)

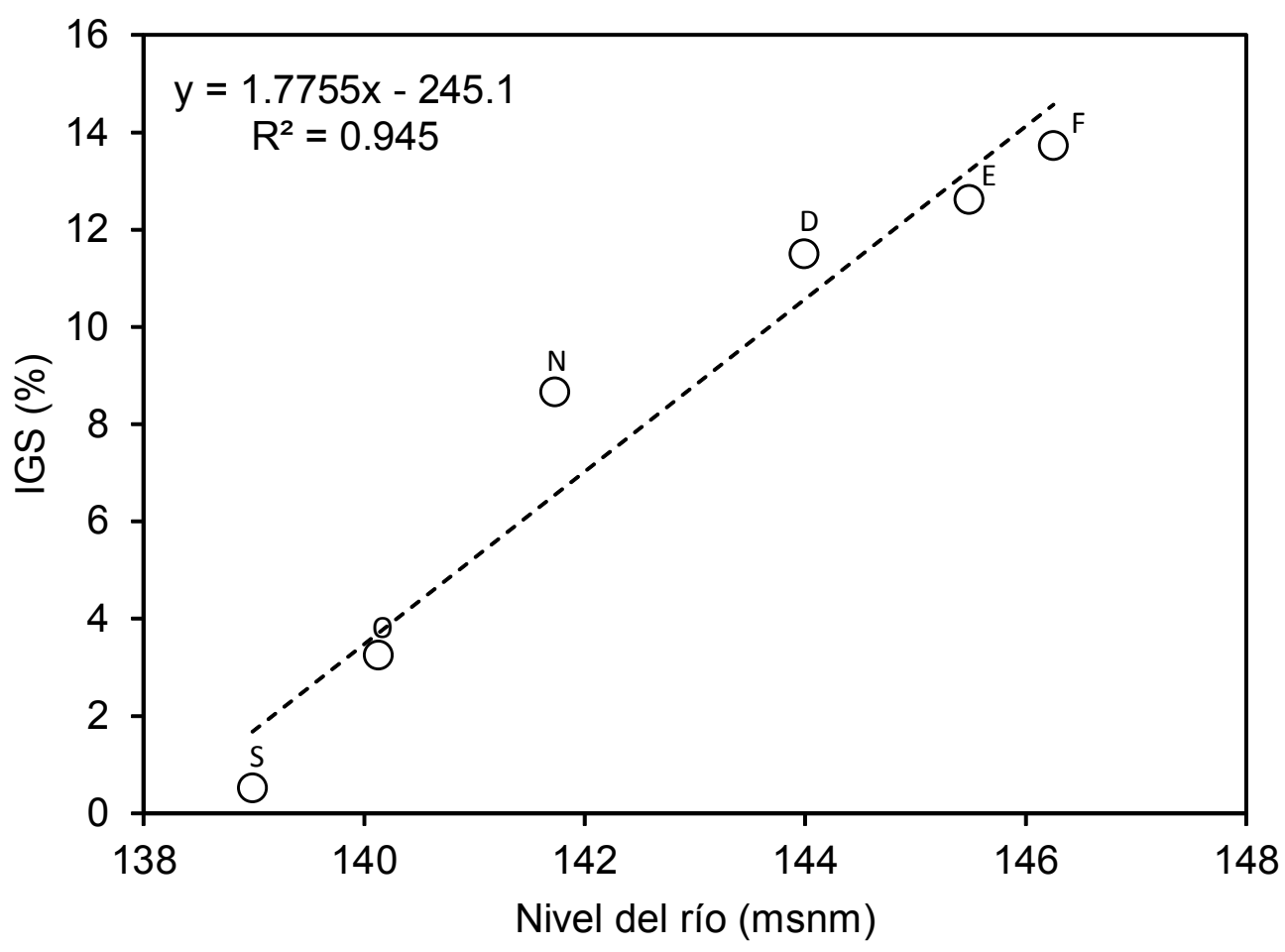

Figura 4. Relación lineal entre el índice gonadosomático (IGS) de hembras de Potamorhina altamazonica y los niveles de agua del río Ucayali (2008-2012). Las letras corresponden a los meses (E: enero; F: febrero; S: setiembre; O: octubre; N: noviembre; D: diciembre) 


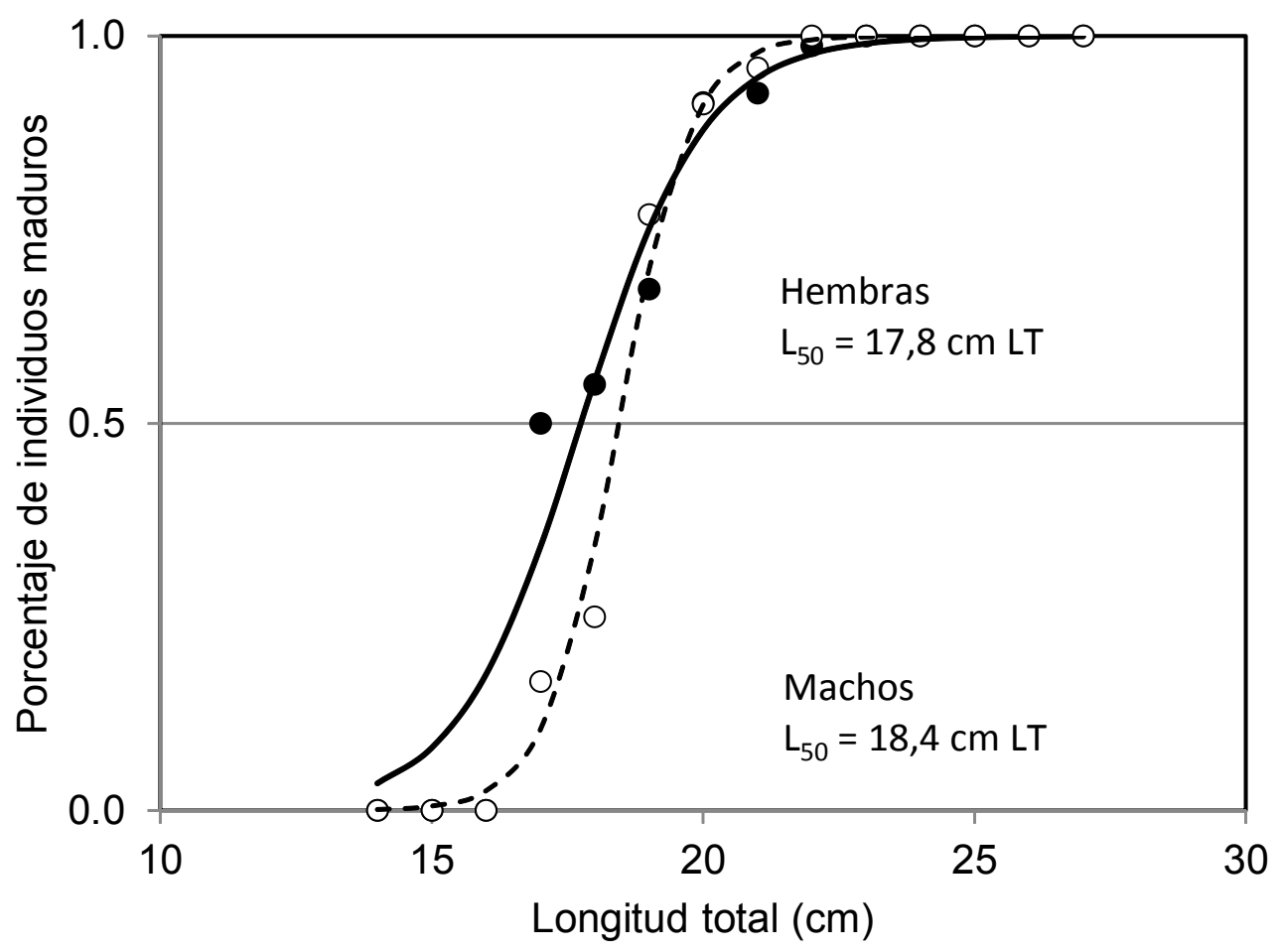

Figura 5. Talla de primera madurez sexual $\left(\mathrm{L}_{50}\right)$ de Potamorhina altamazonica de la región Ucayali. En hembras, $(\bullet)$ es la $\mathrm{L}_{50}$ observada y (-) es la $\mathrm{L}_{50}$ ajustada. En machos, (O) es la $\mathrm{L}_{50}$ observada y (---) la $\mathrm{L}_{50}$ ajustada. Datos de 2008 y 2012

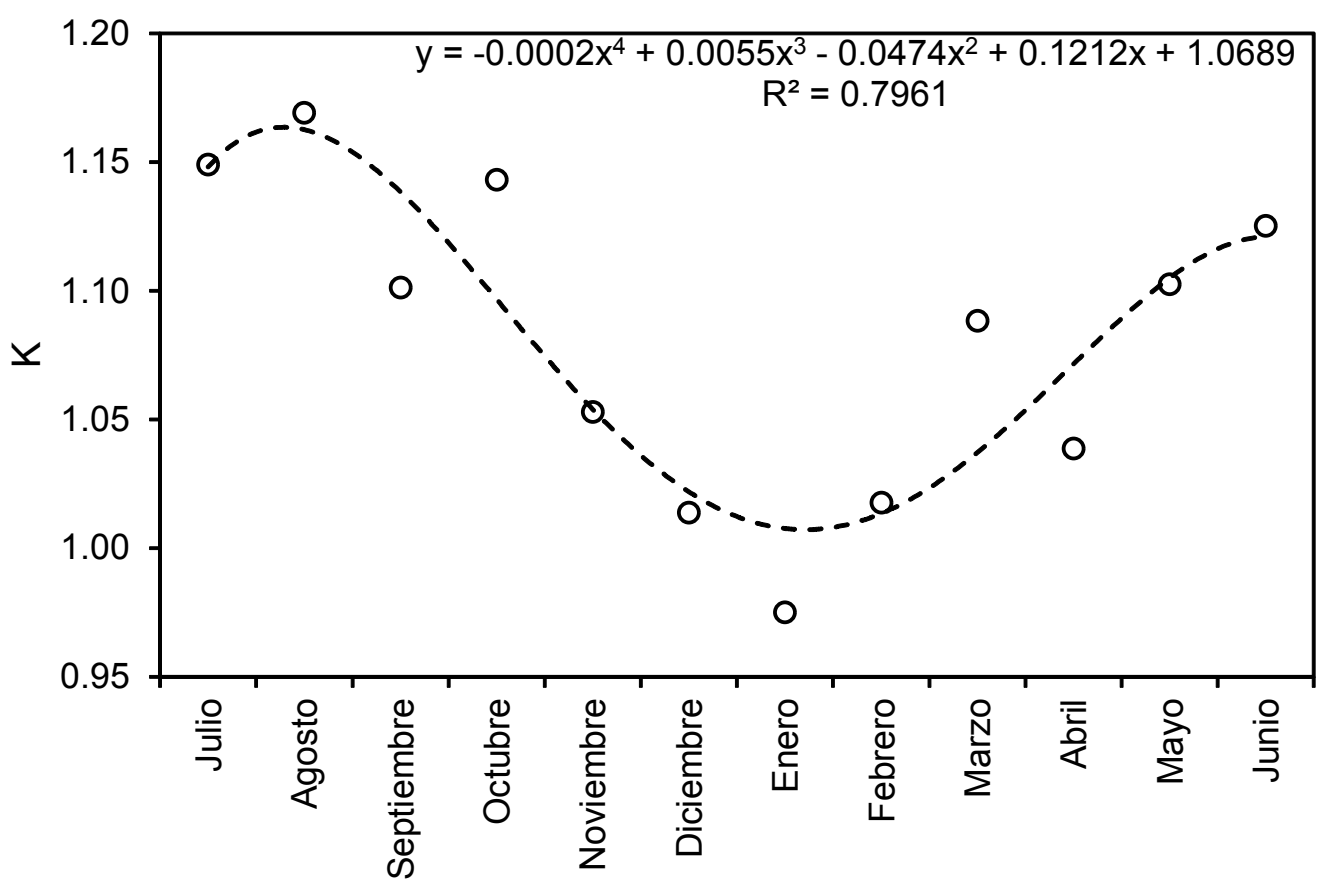

Figura 6. Variación mensual del factor de condición $(\mathrm{K})$ de hembras de Potamorhina altamazonica (2008-2012). (O) corresponde al promedio mensual de K y (---) a la línea de tendencia 
y febrero, donde el coeficiente de determinación fue altamente significativo $\left(\mathrm{r}^{2}=\right.$ 0.945).

\section{Talla Media de Primera Madurez}

La talla media de primera madurez (considerando LT) fue estimada en $\mathrm{L}_{50}=17.8 \mathrm{~cm}$ en hembras y $18.4 \mathrm{~cm}$ en machos. Se observó que las hembras maduran a una talla menor que los machos, aunque sin diferencia estadística (Fig. 5).

\section{Factor de Condición}

El análisis de la variación mensual del factor de condición (K) en llambina permitió observar que las hembras poseen mejor condición somática entre los meses de marzo y noviembre (Fig. 6), donde los valores de $\mathrm{K}$ superaron 1.04; mientras que los valores más bajos se observaron entre diciembre y febrero.

\section{Discusión}

La época de desove de $P$. altamazonica en la región Ucayali puede tener una extensión de hasta siete meses. Se inicia en la época de transición a creciente (octubre), alcanza el máximo durante la época de creciente (principalmente en enero y febrero) y culmina en marzo. Los resultados concuerdan con otros autores (Riofrío, 1993; Aguilar et al., 2009); asimismo, Deza y Bazán (2007) señalan que la reproducción se inicia en setiembre, sin mencionar otros detalles. Por otro lado, en la región Loreto, el proceso dura seis meses: inicia en noviembre y culmina en abril, con el máximo entre diciembre y febrero (García et al., 2010); lo cual evidencia que la época de desove ocurre en forma paralela, tanto en el río Ucayali como en el Amazonas.

La relación positiva entre el IGS y el nivel del río permite observar que el inicio de la madurez gonadal está modulada a los marcados cambios hidrológicos estacionales, sien- do fundamental el periodo de creciente (Welcomme, 1979; Ruffino e Isaac, 1995; Duponchelle et al., 2007), donde el agua es más abundante y de mejor calidad, ya sea tanto para los progenitores como para los descendientes (Lowe-McConnell, 1987; Granado-Lorencio, 1996). Machado-Allison (1994) señala que los cambios físico-químicos del agua ( $\mathrm{pH}$, transparencia, conductividad, concentración de oxígeno y temperatura) durante la creciente favorecen la producción de fitoplancton y zooplancton, esencial para las fases iniciales de vida de los peces. Los bosques de la llanura amazónica se inundan por el desborde del río y se convierten en zonas favorables para la alimentación de la nueva generación de peces (Welcomme, 1985; Junk et al., 1997).

Los machos presentaron dominancia en número, respecto a las hembras, al inicio de la época reproductiva (periodo de transición a creciente), y las hembras lo hicieron en el periodo de creciente, cuando la reproducción es intensa. Las escasas publicaciones sobre la especie en estudio no permiten generar mayor discusión; sin embargo, Muñoz y Van Damme (1999) observaron algunas diferencias en la proporción de sexos, a favor de las hembras en Colossoma macropomun, en el río Ichilo, durante el periodo de reproducción.

Son contados los trabajos de investigación donde se efectuaron estimaciones sobre la talla media de primera madurez $\left(\mathrm{L}_{50}\right)$. Para la región Ucayali, Riofrío (1993) señala que las hembras alcanzan la madurez sexual a los $22.7 \mathrm{~cm}$, mientras que Deza y Bazán (2007) lo encuentran a los $21.6 \mathrm{~cm}$ en hembras y a $20.9 \mathrm{~cm}$ en machos. García y Montreuil (2004), en la región Loreto, la reportan en $21.2 \mathrm{~cm}$ en hembras y $20.8 \mathrm{~cm}$ en machos, y García et al. (2010) en $20.1 \mathrm{~cm}$ en hembras y $19.1 \mathrm{~cm}$ en machos. En el presente estudio se determinó una $\mathrm{L}_{50}$ de $17.8 \mathrm{~cm}$ en hembras y de $18.4 \mathrm{~cm}$ en machos.

Las estimaciones de $\mathrm{L}_{50}$ en la presente investigación difieren de las obtenidas por estos autores (Riofrío, 1993; García y 
Montreuil, 2004; Deza y Bazán, 2007), posiblemente debido a la metodología aplicada, dado que estos emplearon el método de frecuencia acumulada de ejemplares maduros a cada longitud; en tanto que en este trabajo se calculó la proporción de ejemplares maduros respecto al total de especímenes (maduros más inmaduros) en cada talla observada, y se ajustaron mediante una ecuación logística.

Comparando la estimación de $\mathrm{L}_{50}$ de García et al. (2010), quienes hicieron una mejor aplicación de la metodología de estimación, las diferencias de $\mathrm{L}_{50}$ estimadas indicarían que existe una mayor presión de pesca sobre el recurso en la región Ucayali. Diversas investigaciones han evidenciado que la explotación pesquera de un recurso induce a que la especie reduzca su tamaño de primera madurez sexual, con el fin de asegurar el mantenimiento de su población (Murphy, 1968; Hutchings y Myers, 1994; Anderson et al., 2008). Este mecanismo parece haber adoptado el stock llambina que se encuentra en el río Ucayali.

En la Amazonia de Brasil, SánchezBotero y Araujo-Lima (2001) estimaron $\mathrm{L}_{50}$ en $17.03 \mathrm{~cm}$ en hembras para el río Amazonas, en tanto que Amadio y Bittencourt (2005) estimaron $14.82 \mathrm{~cm}$ para hembras en el lago Catalao, Brasil. Nikolsky (1963) y Babiker e Ibrahim (1979) indican que la diferencia de $\mathrm{L}_{50}$ puede estar influenciada por la abundancia y disponibilidad estacional del alimento, la temperatura, el fotoperiodo y factores ambientales de cada localidad; aspectos que explicarían la diferencia entre la estimación de este estudio y los determinados en la otras regiones de la amazonia.

El análisis de la variación temporal del factor de condición mostró que llambina presentó buena condición en los meses de aguas bajas o época seca, principalmente entre julio y octubre; lo que evidencia que el consumo de alimentos de esta especie - que es detritívora- no se encuentra limitado por la reducción del espacio acuático por disminu- ción de las precipitaciones y consecuente disminución del nivel del agua, que sí afecta a las especies omnívoras. Al respecto, YossaPerdomo y Araujo-Lima et al. (1996) encontraron que el detritus es de mejor calidad en la época seca del año; asimismo, Mortillaro et al. (2011) encuentran una alta concentración de ácidos grasos en la materia orgánica particulada suspendida en el agua de los ríos de la Amazonia baja en la época seca.

Winemiller (1989) y Fabré y Vandick (2011) clasifican tres grupos de peces, según sus estrategias de vida: los estacionales o periódicos $(\mathrm{r} 2)$, en equilibrio ( $\mathrm{k}$ ) y oportunistas $(\mathrm{r} 1)$. Los peces estacionales son de rápido crecimiento y alcanzan la madurez sexual a una edad temprana, poseen alta fecundidad y presentan desove total sincronizado al régimen de precipitaciones o de inundación, generalmente en aguas bien oxigenadas (Winemiller y Rose, 1992; Ruffino y Issac, 1995; Godinho et al., 2010). Según los resultados de esta investigación, $P$. altamazonica es una especie estacional o periódica (r2), cuyo desarrollo reproductivo está estrechamente relacionado con los cambios hidrológico-ambientales estacionales.

\section{Agradecimientos}

El autor agradece al Instituto del Mar del Perú (IMARPE) por el financiamiento y logística brindada para el desarrollo de esta investigación. A Fabrice Duponchelle del Institut de Recherche pour le Développement (IRD) y a Julio Valdivia del IMARPE por sus asesorías a lo largo de este trabajo.

\section{Literatura Citada}

1. Aguilar PG, Gallo M, Salas A, Barriga $M$. 2009. Información nutricional sobre algunos peces comerciales de la Amazonia peruana. Lima: Bol Investig Instituto Tecnológico Pesquero del Perú. $65 \mathrm{p}$. 
2. Amadio A, Bittencourt M. 2005. Táticas reprodutivas de peixes em ambientes de várzea na Amazonia Central. En: Biología de las poblaciones de peces de la Amazonía y piscicultura. Comunicaciones del Coloquio Internacional sobre Biología de las Poblaciones de Peces de la Amazonía y Piscicultura. Iquitos, Perú. p 65-75.

3. Anderson $C$, Hsieh $C$, Sandin SA, Hewitt R, Hollowed A, Beddington J, et al. 2008. Why fishing magnifies fluctuations in fish abundance. Nature: 452: 835-839. doi: 10.1038/nature06851

4. Babiker MM, Ibrahim H. 1979. Studies on the biology of reproduction in the cichlid Tilapia nilotica (L): Gonadal maturation and fecundity. J Fish Biol 14: 437-447. doi: 10.1111/j.1095-8649. 1979.tb03541.x

5. Buitrón B, Perea A, Mori J, Sánchez J, Roque C. 2011. Protocolo para estudios sobre el proceso reproductivo de peces pelágicos y demersales. Inf Inst Mar Perú 38: 373-384.

6. Cubillos L. 2005. Biología pesquera y evaluación de stock. Laboratorio de Poblaciones Marinas y Análisis de Pesquerías, Departamento de Oceanografía, UDEC. Concepción, Chile. 198 p.

7. Deza S, Bazán R. 2007. Propuesta de manejo de poblaciones naturales de «llambina» Potamorhina altamazonica y «boquichico» Prochilodus nigricans para la región Ucayali. Proyecto Evaluación para el Manejo de Recursos Pesqueros Amazónicos. Instituto de Investigaciones de la Amazonía Peruana. Iquitos, Perú. $17 \mathrm{p}$.

8. Duponchelle F, Lino F, Hubert $N$, Panfili J, Renno JF, Baras E, et al. 2007. Environment-related life history trait variations of the red-bellied piranha, Pygocentrus nattereri, in two river basins of the Bolivian Amazon. J Fish Biol 71: 1113-1134. doi: 10.1111/j.10958649.2007.01583.x

9. Flores VS. 2013. Influencia de las variaciones estacionales del nivel de agua sobre la reproducción y el crecimiento de Potamorhina altamazonica (Cope, 1879) en el río Ucayali, Perú. Tesis de Maestría. Lima: Univ Nacional Mayor de San Marcos. 58 p.

10. Fabré NN, Vanadick B. 2011. Estratégias de vida e conservaçao das espécies de peixes. En: Nuñez J (ed). Biología de las poblaciones de peces amazónicos y piscicultura. II Coloquio de la red de investigaciones sobre la ictiofauna amazónica (RIIA). Manaus, Brasil. p 73-79.

11. García A, Montreiul V. 2004. Utilización de la talla de primera maduración de llambina (Potamorhina altamazonica, COPE 1878) en la regulación de la explotación de sus poblaciones en la Amazonía peruana. En: Bodmer R, Puertas P, Antunez M (eds). Memoria VI Congreso sobre Manejo de Fauna Silvestre en la Amazonía y Latinoamérica. Iquitos, Perú.

12. García A, Vargas G, Rodríguez $R$, Montreuil V, Ismiño R, Sánchez $H$, et al. 2010a. Aspectos biológicos pesqueros de Potamorhina altamazonica llambina (Cope, 1878) en la región Loreto-Amazonía peruana. Folia Amazónica 19 (1-2): 23-28.

13. García A, Alonso JC, Carvajal F, Moreau J, Nuñez J, Renno JF, et al. 2010b. Life-history characteristics of the large Amazonian migratory catfish Brachyplatystoma rousseauxii in the Iquitos region, Peru. J Fish Biol 75: 25272551. doi: $10.1111 / j .1095-8649.2009$.02444.x

14. Godinho AL, Reis I, Godinho H. 2010. Reproductive ecology of Brazilian freshwater fishes. Environ Biol Fish 87: 143-162. doi: 10.1007/s10641-009-9574-4

15. Guerra H. 1995. Estado actual del conocimiento de la pesquería en la Amazonia Peruana. Instituto de Investigaciones de la Amazonia Peruana-IIAP. Documento Técnico N. ${ }^{\circ} 11$. Iquitos, Perú. $53 \mathrm{p}$.

16. Guerrero HY, Cardillo E, Poleo G, Marcano D. 2009. Reproductive biology of freshwater fishes from the 
Venezuelan floodplains. Fish Physiol Biochem 35: 189-196. doi: 10.1007/ s10695-008-9249-7

17. Granado-Lorencio C. 1996. Ecología de peces. Sevilla, España: Universidad de Sevilla. $353 \mathrm{p}$.

18. Hutchings JA, Myers R. A. 1994. The evolution of alternative mating strategies in variable environments. Evol Ecol 8: 256-268.

19. Junk WJ, Soares MGM, Saint-Paul U. 1997. The Fish. En: Junk WJ (ed). The central Amazon floodplain: ecology of a pulsing system. Berlin: Springer-Verlag. $525 \mathrm{p}$.

20. Lima AC, Araújo-Lima C. 2004. The distributions of larval and juvenile fishes in Amazonian rivers of different nutrient status. Freshwater Biol 49: 787-800. doi: 10.1111/j.1365-2427.2004.01228.x

21. Lowe-McConnell RH. 1987. Ecological studies in tropical fish communities. UK: Cambridge University Press. $382 \mathrm{p}$.

22. Machado-Allison A. 1994. Factors affecting fish communities in the flooded plains of Venezuela. Acta Biol Venezuela 15: 59-75.

23. Mortillaro JM, Abril G, Moreira-Turcq P, Sobrinho RL, Pérez M, Meziane T. 2011. Fatty acid and stable isotope $(\delta 13$ $\mathrm{C}, \delta 15 \mathrm{~N}$ ) signatures of particule organic matter in the lower Amazon River: seasonal contrasts and connectivity between floodplain lakes and the mainstem. Org Geochem 42: 1159-1168. doi: 10.1016/j.orggeochem.2011.08.011

24. Мийоz H, Van Damme P. 1998. Parámetros de reproducción de 4 especies de peces comerciales (Pseudoplatystoma fasciatum, P. trigrinum, Colossoma macropomum y Piractus brachypomun) en la cuenca del río Ichilo (Bolivia). Rev Bol Ecol 4: 39-54.

25. Murphy GI. 1968. Pattern in life history and the environment. Am Nat 102: 391403.
26. Nikolsky DV. 1963. The ecology of fishes. New York, USA: Academic Press. 352 p.

27. Pouilly M, Yunoki T, Rosales C, Torres L. 2004. Trophic structure of fish assemblages from Mamoré River floodplain lakes (Bolivia). Ecol Freshwater Fish 13: 245-257. doi: 10.1111/j.1600-0633.2004.00055.x

28. Riofrío JC. 1993. Informe técnico de proyecto: evaluación de recursos hidrobiológicos en Ucayali. Convenio: IVITA-DIREPE, Pucallpa. Perú. 62 p.

29. Riofrío C, Ferré W, Velarde D. 2008. Contribuciones para el manejo de la pesquería comercial en Pucallpa (Ucayali Perú). En: Pinedo D, Soria C (eds). El manejo de las pesquerías en la Amazonía. Bogotá, Colombia: Mayol Ed. 492 p.

30. Ruffino ML, Isaac VJ. 1995. Life cycle and biological parameters of several Brazilian Amazon fish species. Naga 18(4): 41-45.

31. Sánchez-Botero JI, Araújo-Lima CARM. 2001. As macrófitas aquáticas como berçário para a ictiofauna da várzea do rio Amazonas. Acta Amazonica 31:437-447.

32. Vazzoler AE. 1996. Biología da reproduçao de peixes teleósteos: teoria e prática. Brasil: EDUEM. 169 p.

33. Villamil-Moreno L, Arias-Castellanos A. 2011. Fecundidad de Otocinclus spectabilis. Orinoquia 15(1): 41-47.

34. Welcomme RL. 1979. Fisheries ecology of floodplain rivers. London: Longman. $317 \mathrm{p}$.

35. Welcomme RL. 1985. River Fisheries. Fisheries Technical Paper $\mathrm{N}^{\circ} 262$. Rome: FAO. $330 \mathrm{p}$.

36. Winemiller KO, Rose KA. 1992. Patterns of life-history diversification in North American fishes: implications for population regulation. Can J Fish Aquat Sci 49: 2196-2218. 
37. Winemiller KO. 1989. Patterns of variation in life history among South American fishes in seasonal environments. Oecologia 81: 225-241.

38. Yamamoto KC. 2004. A estrutura de comunidades de peixes em lagos manejados da Amazônia Central. Dissertação de Mestrado. Manaus, Brasil: Instituto
Nacional de Pesquisas da Amazônia/ Universidade Federal do Amazonas. $71 \mathrm{p}$. 39. Yossa-Perdomo M, Araujo-Lima C. 1996. The quality of the detritus used by Amazonian fish during the low water season. En: Biology of tropical fishes. Brasil: INPA. p 81-84. 